\title{
Inverse Problems for the Quadratic Pencil of the Sturm-Liouville Equations with Impulse
}

\author{
Rauf Kh. Amurov ${ }^{1}$ and A. Adiloglu Nabiev² \\ ${ }^{1}$ Department of Mathematics, Faculty of Sciences, Cumhuriyet University, 58140 Sivas, Turkey \\ ${ }^{2}$ Department of Secondary Science and Mathematics Education, Faculty of Education, Cumhuriyet University, 58140 Sivas, Turkey \\ Correspondence should be addressed to Rauf Kh. Amırov; emirov@cumhuriyet.edu.tr
}

Received 22 November 2012; Revised 2 March 2013; Accepted 4 March 2013

Academic Editor: Juan J. Nieto

Copyright (c) 2013 R. Kh. Amırov and A. A. Nabiev. This is an open access article distributed under the Creative Commons Attribution License, which permits unrestricted use, distribution, and reproduction in any medium, provided the original work is properly cited.

\begin{abstract}
In this study some inverse problems for a boundary value problem generated with a quadratic pencil of Sturm-Liouville equations with impulse on a finite interval are considered. Some useful integral representations for the linearly independent solutions of a quadratic pencil of Sturm-Liouville equation have been derived and using these, important spectral properties of the boundary value problem are investigated; the asymptotic formulas for eigenvalues, eigenfunctions, and normalizing numbers are obtained. The uniqueness theorems for the inverse problems of reconstruction of the boundary value problem from the Weyl function, from the spectral data, and from two spectra are proved.
\end{abstract}

\section{Introduction}

The theory of inverse problems for differential operators occupies an important position in the current developments of the spectral theory of linear operators. Inverse problems of spectral analysis consist in the recovery of operators from their spectral data. One takes for the main spectral data, for instance, one, two, or more spectra, the spectral function, the spectrum, and the normalizing constants, the Weyl function. Different statements of inverse problems are possible depending on the selected spectral data. The already existing literature on the theory of inverse problems of spectral analysis is abundant. The most comprehensive account of the current state of this theory and its applications can be found in the monographs of Marchenko [1], Levitan [2], Beals et al. [3], and Yurko [4].

In the present work we consider some inverse problems for the boundary value problem generated by the differential equation

$$
\begin{array}{r}
L_{\lambda} y:=y^{\prime \prime}+\left[\lambda^{2}-2 \lambda p(x)-q(x)\right] y=0, \\
x \in[0, a) \cup(a, \pi]
\end{array}
$$

with the boundary conditions

$$
U(y):=y^{\prime}(0)=0, \quad V(y):=y(\pi)=0
$$

and with the jump conditions

$$
y(a+0)=\alpha y(a-0), \quad y^{\prime}(a+0)=\alpha^{-1} y^{\prime}(a-0),
$$

where $\lambda$ is the spectral parameter, $p(x) \in W_{2}^{1}[0, \pi], q(x) \in$ $L_{2}[0, \pi]$ are real functions, $\alpha$ is a real number, and $\alpha>$ $0, \alpha \neq 1, a \in(\pi / 2, \pi)$. Here we denote by $W_{2}^{m}[0, \pi]$ the space of functions $f(x), x \in[0, \pi]$, such that the derivatives $f^{(m)}(x)(m=\overline{0, n-1})$ are absolutely continuous and $f^{(n)}(x) \epsilon$ $L_{2}[0, \pi]$.

There exist many papers containing a fairly comprehensive analysis of direct and inverse problems of spectral analysis of the Sturm-Liouville equation

$$
l y:=-y^{\prime \prime}+q(x) y=\lambda^{2} y,
$$

a special case $(p(x) \equiv 0)$ of (1). For instance, inverse problems for a regular Sturm-Liouville operator with separated boundary conditions have been investigated in [5] (see also [1-4]).

Some versions of inverse problems for (1) which is a natural generalization of the Sturm-Liouville equation were 
fully studied in [6-14]. Namely, the inverse problems for a pencil $L_{\lambda}$ on the half axis and the entire axis were considered in [6-8], where the scattering data, the spectral function, and the Weyl function, respectively, were taken for the spectral data. The problem of the recovery of (1) from the spectra of two boundary value problems with certain separated boundary conditions was solved in [9]. The analysis of inverse spectral problems for (1) with other kinds of separated boundary conditions as well as with periodic and antiperiodic boundary conditions was the subject of [10] (see also [11]) where the corresponding results of the monograph [1] were extended to the case $p(x) \neq 0$. The inverse periodic problem for the pencil $L_{\lambda}$ was solved in [12] using another approach. We also point out the paper [14], in which the uniqueness of the recovery of the pencil $L_{\lambda}$ from three spectra was investigated.

Boundary value problems with discontinuities inside the interval often appear in mathematics, physics, and other fields of natural sciences. The inverse problems of reconstructing the material properties of a medium from data collected outside of the medium give solutions to many important problems in engineering and geosciences. For example, in electronics, the problem of constructing parameters of heterogeneous electronic lines is reduced to a discontinuous inverse problem $[15,16]$. The reduced mathematical model exhibits the boundary value problem for the equation of type (1) with given spectral information which is described by the desirable amplitude and phase characteristics. Note that the problem of reconstructing the permittivity and conductivity profiles of a one-dimensional discontinuous medium is also closed to the spectral information [17, 18]. Geophysical models for oscillations of the Earth are also reduced to boundary value problems with discontinuity in an interior point [19].

Direct and inverse spectral problems for differential operators without discontinuities have been extensively studied by many authors [20-25]. Some classes of direct and inverse problems for discontinuous boundary value problems in various statements have been considered in $[18,26-32]$ and other works. Boundary value problems with singularity have been studied in [33-37], and for further discussion see the references therein. Note that the inverse spectral problem for the boundary problem (1)-(3) has never been considered before.

In what follows we denote the boundary value problem (1)-(3) by $L(a, \alpha)$. In Section 2 we derive some integral representations for the linearly independent solutions of (1), and using these, we investigate important spectral properties of the boundary value problem $L(a, \alpha)$. In Section 3 the asymptotic formulas for eigenvalues, eigenfunctions, and normalizing numbers of $L(a, \alpha)$ are obtained. Finally, in Section 4 three inverse problems of reconstructing the boundary value problem $L(\alpha, a)$ from the Weyl function, from the spectral data, and from two spectra are considered and the uniqueness theorems are proved.

\section{Integral Representations of Solutions and the Spectral Characteristics}

Let $f_{v}(x, \lambda)(\nu=1,2)$ be solution of (1) under the initial condition

$$
f_{\nu}(0, \lambda)=1, \quad f_{\nu}^{\prime}(0, \lambda)=\lambda w_{\nu}
$$

and discontinuity conditions (3), where $w_{\nu}=(-1)^{\nu+1} i$.

It is obvious that the functions $f_{\nu}(x, \lambda)$ satisfy the integral equations

$$
\begin{aligned}
f_{v}(x, \lambda) & \\
= & l^{+}(x) e^{\lambda w_{\nu} x}+l^{-}(x) e^{\lambda w_{\nu}(2 a-x)} \\
& +l^{+}(x) \int_{0}^{a} \frac{\sin \lambda(x-t)}{\lambda}\{q(t)+2 \lambda p(t)\} f_{\nu}(t, \lambda) d t \\
& -l^{-}(x) \int_{0}^{a} \frac{\sin \lambda(x+t-2 a)}{\lambda}\{q(t)+2 \lambda p(t)\} f_{v}(t, \lambda) d t \\
& +\int_{a}^{x} \frac{\sin \lambda(x-t)}{\lambda}\{q(t)+2 \lambda p(t)\} f_{\nu}(t, \lambda) d t,
\end{aligned}
$$

where $l^{ \pm}(x)=(1 / 2)(l(x) \pm(1 / l(x))$ and

$$
l(x)= \begin{cases}1, & 0 \leq x<a \\ \alpha, & a<x \leq \pi\end{cases}
$$

Using the integral equations (6) and standard successive approximation methods $[7,9,11]$, the following theorem is proved.

Theorem 1. If $q(x) \in L_{2}[-b, b], p(x) \in W_{2}^{1}[-b, b](0<b<$ $\pi)$, then the solution $f_{v}(x, \lambda)$ has the form

$$
f_{v}(x, \lambda)=f_{0 v}(x, \lambda)+\int_{-x}^{x} A_{\nu}(x, t) e^{\lambda w_{\nu} t} d t,
$$

where

$$
\begin{gathered}
f_{0 \nu}(x, \lambda)=l^{+}(x) e^{\lambda w_{\nu} x} R_{\nu}^{+}(x)+l^{-}(x) e^{\lambda w_{\nu}(2 a-x)} R_{\nu}^{-}(x), \\
R_{\nu}^{ \pm}(x)=e^{\mp w_{\nu} \beta^{ \pm}(x)}, \quad \beta^{ \pm}(x)=\int_{(a \mp a) / 2}^{x} p(t) d t,
\end{gathered}
$$

and the function $A_{v}(x, t)$ satisfies the inequality

$$
\int_{-x}^{x}\left|A_{\nu}(x, t)\right| d t \leq e^{c \sigma(x)}-1
$$

with

$$
\begin{aligned}
& \sigma(x)=\int_{0}^{x}[(x-t)|q(t)|+2|p(t)|] d t, \\
& c=2\left(\alpha^{+}+\left|\alpha^{-}\right|+1\right), \quad \alpha^{ \pm}=\frac{1}{2}\left(\alpha \pm \frac{1}{\alpha}\right) .
\end{aligned}
$$


Let $\varphi(x, \lambda)$ be the solution of (1) that satisfies the initial conditions

$$
\varphi(0, \lambda)=1, \quad \varphi^{\prime}(0, \lambda)=0,
$$

and the jump condition (3).

Then by using Theorem 1 , we can formulate the following assertion.

Theorem 2. Let $q(x) \in L_{2}[0, \pi], p(x) \in W_{2}^{1}[0, \pi]$. Then there are the functions $A(x, t), B(x, t)$ whose first order partial derivatives are summable on $[0, \pi]$ for each $x \in[0, \pi]$ such that the representation

$$
\varphi(x, \lambda)=\varphi_{0}(x, \lambda)+\int_{0}^{x} A(x, t) \cos \lambda t d t+\int_{0}^{x} B(x, t) \sin \lambda t d t
$$

is satisfied, where

$$
\begin{aligned}
\varphi_{0}(x, \lambda)= & l^{+}(x) \cos \left[\lambda x-\beta^{+}(x)\right] \\
& +l^{-}(x) \cos \left[\lambda(2 a-x)-\beta^{-}(x)\right] .
\end{aligned}
$$

Moreover, the relations

$$
\begin{aligned}
\alpha^{+} \beta^{+}(x) & =\alpha^{+} x p(0) \\
& +2 \int_{0}^{x}\left[A(\xi, \xi) \sin \beta^{+}(\xi)-B(\xi, \xi) \cos \beta^{+}(\xi)\right] d \xi \\
2 \frac{d}{d x}\left[A(x, x) \cos \beta^{+}(x)+B(x, x) \sin \beta^{+}(x)\right] & \\
= & \alpha^{+}\left[q(x)+p^{2}(x)\right] \\
\left.2 \frac{d}{d x}\left[A(x, t) \cos \beta^{-}(x)-B(x, t) \sin \beta^{-}(x)\right]\right|_{t=2 a-x-0} ^{t=2 a-x+0} & \\
= & \alpha^{-}\left[q(x)+p^{2}(x)\right] \\
\left.A_{t}(x, t)\right|_{t=0} & =B(x, 0)=0
\end{aligned}
$$

are held.

If one additionally supposes that $q(x) \in W_{2}^{1}[0, \pi], p(x) \in$ $W_{2}^{2}[0, \pi]$, then the functions $A(x, t)$ and $B(x, t)$ satisfy the system of partial differential equations

$$
\begin{aligned}
& A_{x x}(x, t)-q(x) A(x, t)-2 p(x) B_{t}(x, t)=A_{t t}(x, t), \\
& B_{x x}(x, t)-q(x) B(x, t)+2 p(x) A_{t}(x, t)=B_{t t}(x, t) .
\end{aligned}
$$

Conversely, if the second order derivatives of functions $A(x, t), B(x, t)$ are summable on $[0, \pi]$ for each $x \in[0, \pi]$ and $A(x, t), B(x, t)$ satisfy equalities (16) and conditions (15), then the function $\varphi(x, \lambda)$ which is defined by $(13)$ is a solution of (1) satisfying initial conditions (12) and discontinuity conditions (3).

One here supposes that the function $q(x)$ satisfies the additional condition

$$
\int_{0}^{\pi}\left\{\left|y^{\prime}(x)\right|^{2}+q(x)|y(x)|^{2}\right\} d x>0
$$

for all $y(x) \in W_{2}^{2}[0, a) \cup(a, \pi]$ such that $y(x) \neq 0$ and

$$
y^{\prime}(0) \overline{y(0)}-y^{\prime}(\pi) \overline{y(\pi)}=0 .
$$

Definition 3. A complex number $\lambda_{0}$ is called an eigenvalue of the boundary value problem $L(\alpha, a)$ if (1) with $\lambda=\lambda_{0}$ has a nontrivial solution $y_{0}(x)$ satisfying the boundary conditions (2) and the jump conditions (3). In this case $y_{0}(x)$ is called the eigenfunction of the problem $L(\alpha, a)$ corresponding to the eigenvalue $\lambda_{0}$. The number of linearly independent solutions of the problem $L(\alpha, a)$ for a given eigenvalue $\lambda_{0}$ is called the multiplicity of $\lambda_{0}$.

The following lemmas can be proved analogously to the corresponding assertions in [11].

Lemma 4. The eigenvalues of the boundary value problem $L(\alpha, a)$ are real, nonzero, and simple.

Proof. We define a linear operator $L_{0}$ in the Hilbert space $L_{2}[0, \pi]$ as follows. The domain $D\left(L_{0}\right)$ consists of all functions $y(x) \in W_{2}^{2}[0, \pi]$ satisfying the boundary conditions (2) and the jump conditions (3). For $y \in D\left(L_{0}\right)$, we set $L_{0} y=-y^{\prime \prime}+q(x) y$. Integration by part yields

$$
\begin{aligned}
\left(L_{0} y, y\right) & =\int_{0}^{\pi} L_{0} y \bar{y}(x) d x \\
& =\int_{0}^{\pi}\left(\left|y^{\prime}(x)\right|^{2}+q(x)|y(x)|^{2}\right) d x .
\end{aligned}
$$

Since condition (17) holds, it follows that $\left(L_{0} y, y\right)>0$.

Let $\lambda_{0}$ be an eigenvalue of the boundary value problem $L(\alpha, a)$ and $y_{0}(x)$ an eigenfunction corresponding to this eigenvalue and normalized by the condition $\left(y_{0}, y_{0}\right)=1$. By taking the inner product of both sides of the relation $y_{0}^{\prime \prime}(x)+\left[\lambda_{0}^{2}-2 \lambda_{0} p(x)-q(x)\right] y_{0}(x)=0$ by $y_{0}(x)$, we obtain $\lambda_{0}^{2}-2 \lambda_{0}\left(p y_{0}, y_{0}\right)-\left(L_{0} y_{0}, y_{0}\right)=0$ and hence

$$
\lambda_{0}=\left(p y_{0}, y_{0}\right) \pm \sqrt{\left(p y_{0}, y_{0}\right)^{2}+\left(L_{0} y_{0}, y_{0}\right)}
$$

The desired assertion follows from the last relation by virtue of $\left(L_{0} y_{0}, y_{0}\right)>0$ with regard to the fact that $p(x)$ is real.

Let us show that $\lambda_{0}$ is a simple eigenvalue. Assume that this is not true. Suppose that $y_{1}(x)$ and $y_{2}(x)$ are linearly independent eigenfunctions corresponding to the eigenvalue $\lambda_{0}$. Then for a given value of $\lambda_{0}$, each solution $y_{0}(x)$ of (1) will be given as linear combination of solutions $y_{1}(x)$ and $y_{2}(x)$. Moreover it will satisfy boundary conditions (2) and discontinuity conditions (3). However, it is impossible.

Lemma 5. The problem (1)-(3) does not have associated functions.

Proof. Let $y_{0}(x)$ be an eigenfunction corresponding to eigenvalue $\lambda_{0}$ and normalized by the condition $\left(y_{0}, y_{0}\right)=1$ of the problem (1)-(3). Suppose that $y_{1}(x)$ is an associated function of eigenfunction $y_{0}(x)$, that is, the following equalities hold:

$$
\begin{gathered}
\lambda_{0}^{2} y_{0}-2 \lambda_{0} p(x) y_{0}-L_{0} y_{0}=0, \\
\lambda_{0}^{2} y_{1}-2 \lambda_{0} p(x) y_{1}-L_{0} y_{1}+2\left(\lambda_{0}-p(x)\right) y_{0}=0 .
\end{gathered}
$$


If these equations are multiplied by $y_{1}(x)$ and $y_{0}(x)$, respectively, as inner product, subtracting them side by side and taking into our account that operator $L_{0}$ is symmetric, the function $p(x)$ and $\lambda_{0}$ are real, we get $\lambda_{0}=\left(p y_{0}, y_{0}\right)$. Due to the condition (6), $\lambda_{0}=\left(p y_{0}, y_{0}\right)$ does not agree with $\left(19^{\prime}\right)$. Therefore, the assertion is not true.

Lemma 6. Eigenfunctions corresponding to different eigenvalues of the problem $L(\alpha, a)$ are orthogonal in the sense of the equality

$$
\left(\lambda_{1}+\lambda_{2}\right)\left(y_{1}, y_{2}\right)-2\left(p y_{1}, y_{2}\right)=0,
$$

where $(\cdot, \cdot)$ denotes the inner product in $L_{2}[0, \pi]$.

Lemma 7. Let $y(x, \lambda)$ be a solution of (1) satisfying the condition (18) and the jump conditions (3). Then $\lambda$ is real and nonzero and

$$
\int_{0}^{\pi}(\lambda-p(x))|y(x, \lambda)|^{2} d x \neq 0 .
$$

Moreover, the sign of the left-hand side of (22) is similar to the sign of $\lambda$.

\section{Properties of the Spectrum}

In this section we investigate some spectral properties of the boundary value problem $L(\alpha, a)$.

Let $\psi(x, \lambda)$ be a solution of (1) with the conditions $\psi(\pi, \lambda)=0, \psi^{\prime}(\pi, \lambda)=1$ and the jump conditions (3). It is clear that function $\psi(x, \lambda)$ is entire in $\lambda$ for each fixed $x$.

Denote $\Delta(\lambda)=\langle\psi(x, \lambda), \varphi(x, \lambda)\rangle$, where $\langle y, z\rangle:=$ $y^{\prime} z-y z^{\prime}$. By virtue of Liouville's formula, the Wronskian $\langle\psi(x, \lambda), \varphi(x, \lambda)\rangle$ does not depend on $x$. The function $\Delta(\lambda)$ is called the characteristic function of $L(\alpha, a)$. Obviously, the function $\Delta(\lambda)$ is entire in $\lambda$ and it has at most a countable set of zeros $\left\{\lambda_{n}\right\}$.

Lemma 8. The zeros $\left\{\lambda_{n}\right\}$ of the characteristic function $\Delta(\lambda)$ coincide with the eigenvalues of the boundary value problem $L(\alpha, a)$. The functions $\varphi\left(x, \lambda_{n}\right)$ and $\psi\left(x, \lambda_{n}\right)$ are eigenfunctions corresponding to the eigenvalue $\lambda_{n}$, and there exists a sequence $\left\{\beta_{n}\right\}$ such that

$$
\psi\left(x, \lambda_{n}\right)=\beta_{n} \varphi\left(x, \lambda_{n}\right), \quad \beta_{n} \neq 0 .
$$

Proof. Let $\Delta\left(\lambda_{0}\right)=0$. Then by virtue of $\left\langle\psi\left(x, \lambda_{0}\right), \varphi\left(x, \lambda_{0}\right)\right\rangle=$ $0, \varphi\left(x, \lambda_{0}\right)=C \psi\left(x, \lambda_{0}\right)$ for some constant $C$. Hence $\lambda_{0}$ is an eigenvalue and $\varphi\left(x, \lambda_{0}\right), \psi\left(x, \lambda_{0}\right)$ are eigenfunctions related to $\lambda_{0}$.

Conversely, let $\lambda_{0}$ be an eigenvalue of $L(\alpha, a)$, show that $\Delta\left(\lambda_{0}\right)=0$. Assuming the converse suppose that $\Delta\left(\lambda_{0}\right) \neq 0$. In this case the functions $\varphi\left(x, \lambda_{0}\right)$ and $\psi\left(x, \lambda_{0}\right)$ are linearly independent. Then $y\left(x, \lambda_{0}\right)=c_{1} \varphi\left(x, \lambda_{0}\right)+c_{2} \psi\left(x, \lambda_{0}\right)$ is a general solution of the problem $L(\alpha, a)$. If $c_{1} \neq 0$, we can write

$$
\varphi\left(x, \lambda_{0}\right)=\frac{1}{c_{1}} y\left(x, \lambda_{0}\right)-\frac{c_{2}}{c_{1}} \psi\left(x, \lambda_{0}\right) .
$$

Then we have

$$
\begin{aligned}
\langle\varphi & \left.\left(x, \lambda_{0}\right), \psi\left(x, \lambda_{0}\right)\right\rangle \\
& =\frac{1}{c_{1}}\left[y^{\prime}\left(\pi, \lambda_{0}\right) \psi\left(\pi, \lambda_{0}\right)-y\left(\pi, \lambda_{0}\right) \psi^{\prime}\left(\pi, \lambda_{0}\right)\right] \\
& =-\frac{1}{c_{1}} y\left(\pi, \lambda_{0}\right) \\
& =0
\end{aligned}
$$

which is a contradiction.

Note that we have also proved that for each eigenvalue there exists only one eigenfunction (up to a multiplicative constant). Therefore there exists sequence $\beta_{n}$ such that $\psi\left(x, \lambda_{n}\right)=\beta_{n} \varphi\left(x, \lambda_{n}\right)$.

Let us denote

$$
\alpha_{n}:=\int_{0}^{\pi} \varphi^{2}\left(x, \lambda_{n}\right) d x-\frac{1}{\lambda_{n}} \int_{0}^{\pi} p(x) \varphi^{2}\left(x, \lambda_{n}\right) d x .
$$

The numbers $\left\{\alpha_{n}\right\}$ are called normalized numbers of the boundary value problem $L(\alpha, a)$.

Lemma 9. The equality $\dot{\Delta}\left(\lambda_{n}\right)=-2 \lambda_{n} \alpha_{n} \beta_{n}$ holds. Here $\dot{\Delta}(\lambda)=(d / d \lambda) \Delta(\lambda)$.

Proof. If we differentiate the equalities

$$
\begin{aligned}
& -\varphi^{\prime \prime}(x, \lambda)+[2 \lambda p(x)+q(x)] \varphi(x, \lambda)=\lambda^{2} \varphi(x, \lambda), \\
& -\psi^{\prime \prime}(x, \lambda)+[2 \lambda p(x)+q(x)] \psi(x, \lambda)=\lambda^{2} \psi(x, \lambda)
\end{aligned}
$$

with respect to $\lambda$, we get

$$
\begin{array}{r}
-\dot{\varphi}^{\prime \prime}(x, \lambda)+[2 \lambda p(x)+q(x)] \dot{\varphi}(x, \lambda) \\
=\lambda^{2} \dot{\varphi}(x, \lambda)+2[\lambda-p(x)] \varphi(x, \lambda), \\
-\dot{\psi}^{\prime \prime}(x, \lambda)+[2 \lambda p(x)+q(x)] \dot{\psi}(x, \lambda) \\
=\lambda^{2} \dot{\psi}(x, \lambda)+2[\lambda-p(x)] \psi(x, \lambda) .
\end{array}
$$

By virtue of these equalities we have

$$
\begin{gathered}
\frac{d}{d x}\left\{\varphi(x, \lambda) \dot{\psi}^{\prime}(x, \lambda)-\varphi^{\prime}(x, \lambda) \dot{\psi}(x, \lambda)\right\} \\
=2[\lambda-p(x)] \varphi(x, \lambda) \psi(x, \lambda), \\
\frac{d}{d x}\left\{\dot{\varphi}(x, \lambda) \psi^{\prime}(x, \lambda)-\dot{\varphi}^{\prime}(x, \lambda) \psi(x, \lambda)\right\} \\
=2[\lambda-p(x)] \varphi(x, \lambda) \psi(x, \lambda) .
\end{gathered}
$$


If the last equations are integrated from $x$ to $\pi$ and from 0 to $x$, respectively, by the discontinuity conditions we obtain

$$
\begin{aligned}
& \left.\left(\varphi(\xi, \lambda) \dot{\psi}^{\prime}(\xi, \lambda)-\varphi^{\prime}(\xi, \lambda) \dot{\psi}(\xi, \lambda)\right)\right|_{x} ^{\pi} \\
& =-2 \int_{x}^{\pi}(\lambda-p(\xi)) \varphi(\xi, \lambda) \psi(\xi, \lambda) d \xi, \\
& \left.\left(\dot{\varphi}(\xi, \lambda) \psi^{\prime}(\xi, \lambda)-\dot{\varphi}^{\prime}(\xi, \lambda) \psi(\xi, \lambda)\right)\right|_{0} ^{x} \\
& \quad=-2 \int_{0}^{x}(\lambda-p(\xi)) \varphi(\xi, \lambda) \psi(\xi, \lambda) d \xi .
\end{aligned}
$$

If we add the last equalities side by side, we get

$$
\begin{gathered}
W[\dot{\varphi}(\xi, \lambda), \psi(\xi, \lambda)]+W[\varphi(\xi, \lambda), \dot{\psi}(\xi, \lambda)] \\
=-2 \int_{0}^{\pi}(\lambda-p(\xi)) \varphi(\xi, \lambda) \psi(\xi, \lambda) d \xi
\end{gathered}
$$

or

$$
\dot{\Delta}(\lambda)=-2 \int_{0}^{\pi}(\lambda-p(\xi)) \varphi(\xi, \lambda) \psi(\xi, \lambda) d \xi
$$

For $\lambda \rightarrow \lambda_{n}$, this yields

$$
\begin{aligned}
\dot{\Delta}\left(\lambda_{n}\right) & =-2 \int_{0}^{\pi}\left(\lambda_{n}-p(\xi)\right) \varphi\left(\xi, \lambda_{n}\right) \psi\left(\xi, \lambda_{n}\right) d \xi \\
& =-2 \beta_{n} \int_{0}^{\pi}\left(\lambda_{n}-p(\xi)\right) \varphi^{2}\left(\xi, \lambda_{n}\right) d \xi \\
& =-2 \lambda_{n} \beta_{n}\left[\int_{0}^{\pi} \varphi^{2}\left(\xi, \lambda_{n}\right) d \xi\right. \\
& \left.-\frac{1}{\lambda_{n}} \int_{0}^{\pi} p(\xi) \varphi^{2}\left(\xi, \lambda_{n}\right) d \xi\right] \\
= & -2 \lambda_{n} \beta_{n} \alpha_{n} .
\end{aligned}
$$

The lemma is proved.

Let $\Delta_{0}(\lambda)=\alpha^{+} \cos \left[\lambda \pi-\beta^{+}(\pi)\right]+\alpha^{-} \cos \left[\lambda(2 a-\pi)+\beta^{-}(\pi)\right]$ and $\left\{\lambda_{n}^{0}\right\}$ are zeros of $\Delta_{0}(\lambda)$.

Lemma 10. The roots of the characteristic equation $\Delta_{0}(\lambda)=0$ are separate, that is

$$
\inf _{n \neq m}\left|\lambda_{n}^{0}-\lambda_{m}^{0}\right|=\beta>0 .
$$

Proof. Let $\lambda \pi-\beta^{+}(\pi)=x$. Then, $\lambda(2 a-\pi)+\beta^{-}(\pi)=k x+b$, where $k=(2 a-\pi) / \pi, b=\beta^{+}(\pi)((2 a-\pi) / \pi)+\beta^{-}(\pi)$. Since $a \in(\pi / 2, \pi)$, then $k \in(0,1)$. Using these notations we can rewrite the equation $\Delta_{0}(\lambda)=0$ in the following form:

$$
A \cos x=\cos (k x+b) .
$$

Here $A=-\left(\alpha^{+} / \alpha^{-}\right)$which implies that $|A|>1$. Preliminarily show that there are no multiple roots of (35). Assuming the converse we suppose $x_{0}$ to be a multiple root of (35). Then

$$
A \sin x_{0}=k \sin \left(k x_{0}+b\right)
$$

holds. Now (35) and (36) imply that $A^{2}=1-\left(1-k^{2}\right) \sin ^{2}\left(k x_{0}+\right.$ $b) \leq 1$ which is a contradiction. Therefore, (35) has no multiple roots.

Further assuming (34) not to be true let $\left\{x_{p}^{\prime}\right\}$ and $\left\{x_{p}^{\prime \prime}\right\}$ be increasing sequences of roots of (35) such that $x_{p}^{\prime} \neq x_{p}^{\prime \prime}$ and

$$
\lim _{p \rightarrow \infty}\left|x_{p}^{\prime}-x_{p}^{\prime \prime}\right|=0 \text {. }
$$

If we assume that $x_{p}^{\prime}=2 n_{p} \pi+r_{p}^{\prime}$, where $n \in \mathbb{N}$ and $\left\{r_{p}^{\prime}\right\}$ is a bounded sequence $\left(0<r_{p}^{\prime}<2 \pi\right)$, then from (37) we find that $x_{p}^{\prime \prime}=2 n_{p} \pi+r_{p}^{\prime \prime}$, where $\left\{r_{p}^{\prime \prime}\right\}$ is a bounded sequence such that $\lim _{p \rightarrow \infty}\left|r_{p}^{\prime}-r_{p}^{\prime \prime}\right|=0$. It is obvious that $k x_{p}^{\prime}=2 \pi\left[k n_{p}\right]+$ $s_{p}^{\prime}, k x_{p}^{\prime \prime}=2 \pi\left[k n_{p}\right]+s_{p}^{\prime \prime}$, where $s_{p}^{\prime}=2 \pi\left\{k n_{p}\right\}+r_{p}^{\prime} k, s_{p}^{\prime \prime}=$ $2 \pi\left\{k n_{p}\right\}+r_{p}^{\prime \prime} k$ and $\lim _{p \rightarrow \infty}\left|s_{p}^{\prime}-s_{p}^{\prime \prime}\right|=0$. Here $[\cdot]$ and $\{\cdot\}$ denote the integer and fractional parts of a real number, respectively. Since sequences $\left\{r_{p}^{\prime}\right\}_{p \geq 1},\left\{r_{p}^{\prime \prime}\right\}_{p \geq 1}\left\{s_{p}^{\prime}\right\}_{p \geq 1}$ and $\left\{s_{p}^{\prime \prime}\right\}_{p \geq 1}$ will be bounded, without loss of generality we can assume that these sequences are convergent. Then let

$$
\begin{aligned}
& \lim _{p \rightarrow \infty} r_{p}^{\prime}=\lim _{p \rightarrow \infty} r_{p}^{\prime \prime}=x_{0}, \\
& \lim _{p \rightarrow \infty} s_{p}^{\prime}=\lim _{p \rightarrow \infty} s_{p}^{\prime \prime}=y_{0} .
\end{aligned}
$$

Therefore, we can write the equality $A \cos x_{p}^{\prime}=\cos \left(k x_{p}^{\prime}+b\right)$ as

$$
A \cos r_{p}^{\prime}=\cos \left(s_{p}^{\prime}+b\right) .
$$

Then by virtue of (38) and (39), from (40) we get

$$
A \cos x_{0}=\cos \left(y_{0}+b\right) \text {. }
$$

Similarly we can obtain

$$
A \cos r_{p}^{\prime \prime}=\cos \left(s_{p}^{\prime \prime}+b\right) \text {. }
$$

Further, from (40) and (42), we have

$$
A \sin \frac{r_{p}^{\prime}+r_{p}^{\prime \prime}}{2} \sin \frac{r_{p}^{\prime}-r_{p}^{\prime \prime}}{2}=\sin \left(\frac{s_{p}^{\prime}+s_{p}^{\prime \prime}}{2}+b\right) \sin \frac{s_{p}^{\prime}-s_{p}^{\prime \prime}}{2} .
$$

Let us write this equality as

$$
\begin{aligned}
A \sin & \frac{r_{p}^{\prime}+r_{p}^{\prime \prime}}{2} \sin \frac{r_{p}^{\prime}-r_{p}^{\prime \prime}}{2} \\
= & \sin \left(\frac{s_{p}^{\prime}+s_{p}^{\prime \prime}}{2}+b\right) \sin \frac{k\left(r_{p}^{\prime}-r_{p}^{\prime \prime}\right)}{2} .
\end{aligned}
$$

Now dividing both sides of equality (44) by $\left(r_{p}^{\prime}-r_{p}^{\prime \prime}\right) / 2 \neq 0$ and taking limit as $p \rightarrow \infty$, by virtue of (3) and (39), we obtain

$$
A \sin x_{0}=k \sin \left(y_{0}+b\right) .
$$

Finally, from (41) and (45), we conclude that $A^{2}=1-(1-$ $\left.k^{2}\right) \sin ^{2}\left(y_{0}+b\right) \leq 1$ which is a contradiction. Hence roots of equation $\Delta_{0}(\lambda)=0$ are separate. The lemma is proved. 
Denote

$$
\begin{aligned}
& \Gamma_{n}=\left\{\lambda:|\lambda|=\left|\lambda_{n}^{0}\right|+\frac{\beta}{2}\right\}, \quad n=0,1, \ldots, \\
& G_{\delta}:=\left\{\lambda:\left|\lambda-\lambda_{n}^{0}\right| \geq \delta\right\} \quad(\delta>0),
\end{aligned}
$$

where $\delta$ is sufficiently small positive number $(\delta \ll \beta / 2)$.

Lemma 11. For sufficiently large values of $n$, one has

$$
\left|\Delta(\lambda)-\Delta_{o}(\lambda)\right|<\frac{C_{\delta}}{2} e^{|\operatorname{Im} \lambda| \pi}, \quad \lambda \in \Gamma_{n} .
$$

Proof. As it is shown in [38], $\left|\Delta_{o}(\lambda)\right| \geq C_{\delta} e^{|\operatorname{Im} \lambda| \pi}$ for all $\lambda \epsilon$ $\overline{G_{\delta}}$, where $C_{\delta}>0$ is some constant. On the other hand, since

$$
\begin{aligned}
& \lim _{|\lambda| \rightarrow \infty} e^{-|\operatorname{Im} \lambda| \pi}\left(\Delta(\lambda)-\Delta_{o}(\lambda)\right) \\
&=\lim _{|\lambda| \rightarrow \infty} e^{-|\operatorname{Im} \lambda| \pi}\left(\int_{0}^{\pi} \widetilde{A}(\pi, t) \cos \lambda t d t\right. \\
&\left.\quad+\int_{0}^{\pi} \widetilde{B}(\pi, t) \sin \lambda t d t\right)
\end{aligned}
$$

$=0$

for sufficiently large values of $n$ (see [1]) we get (47). The lemma is proved.

Lemma 12. The problem $L(\alpha, a)$ has countable set of eigenvalues. If one denotes by $\lambda_{1}, \lambda_{2}, \ldots$ the positive eigenvalues arranged in increasing order and by $\lambda_{-1}, \lambda_{-2}, \ldots$ the negative eigenvalues arranged in decreasing order, then eigenvalues of the problem $L(\alpha, a)$ have the asymptotic behavior

$$
\lambda_{n}=\lambda_{n}^{0}+\frac{d_{n}}{\lambda_{n}^{0}}+\frac{\delta_{n}}{\lambda_{n}^{0}}, \quad n \longrightarrow \pm \infty,
$$

where $\delta_{n} \in l_{2}$ and $d_{n}$ is a bounded sequence, $\lambda_{n}^{0}=n+$ $(1 / \pi) \beta^{+}(\pi)+h_{n}, \sup _{n}\left|h_{n}\right|<\infty$.

Proof. According to Lemma 11, if $n$ is a sufficiently large natural number and $\lambda \in \Gamma_{n}$, we have $\left|\Delta_{o}(\lambda)\right| \geq C_{\delta} e^{|\operatorname{Im} \lambda| \pi}>$ $\left(C_{\delta} / 2\right) e^{|\operatorname{Im} \lambda| \pi}>\left|\Delta(\lambda)-\Delta_{o}(\lambda)\right|$. Applying Rouche's theorem we conclude that for sufficiently large $n$ inside the contour $\Gamma_{n}$ the functions $\Delta_{o}(\lambda)$ and $\Delta_{o}(\lambda)+\left\{\Delta(\lambda)-\Delta_{o}(\lambda)\right\}=\Delta(\lambda)$ have the same number of zeros counting their multiplicities. That is, there are exactly $(n+1)$ zeros $\lambda_{0}, \lambda_{1}, \ldots, \lambda_{n}$. in $\Gamma_{n}$. Analogously, it is shown by Rouche's theorem that, for sufficiently large values of $n$, the function $\Delta(\lambda)$ has a unique zero inside each circle $\left|\lambda-\lambda_{n}^{0}\right|<\delta$. Since $\delta>0$ is arbitrary, it follows that $\lambda_{n}=\lambda_{n}^{0}+\varepsilon_{n}$, where $\lim _{n \rightarrow \infty} \varepsilon_{n}=0$. Further according to $\Delta\left(\lambda_{n}\right)=0$, we have

$$
\begin{gathered}
\Delta_{0}\left(\lambda_{n}^{0}+\varepsilon_{n}\right)+\int_{0}^{\pi} A(\pi, t) \cos \left(\lambda_{n}^{0}+\varepsilon_{n}\right) t d t \\
+\int_{0}^{\pi} B(\pi, t) \sin \left(\lambda_{n}^{0}+\varepsilon_{n}\right) t d t=0 .
\end{gathered}
$$

On the other hand, since

$$
\begin{aligned}
\Delta_{0} & (\lambda) \\
= & \alpha^{+} \cos \left(\lambda \pi-\beta^{+}(\pi)\right)+\alpha^{-} \cos \left(\lambda(2 a-\pi)+\beta^{-}(\pi)\right), \\
& \Delta_{0}\left(\lambda_{n}^{0}+\varepsilon_{n}\right)=\dot{\Delta}_{o}\left(\lambda_{n}^{0}\right) \varepsilon_{n}+o\left(\varepsilon_{n}\right), \quad n \longrightarrow \infty,
\end{aligned}
$$

(50) takes the form of

$$
\begin{aligned}
& \dot{\Delta}_{0}\left(\lambda_{n}^{0}\right) \varepsilon_{n}+\int_{0}^{\pi} A(\pi, t) \cos \left(\lambda_{n}^{0}+\varepsilon_{n}\right) t d t \\
& \quad+\int_{0}^{\pi} B(\pi, t) \sin \left(\lambda_{n}^{0}+\varepsilon_{n}\right) t d t+o\left(\varepsilon_{n}\right)=0, \quad n \longrightarrow \infty
\end{aligned}
$$

It is easy to see that the function $\Delta_{0}(\lambda)$ is type of "Sine" [39], so there exists $\gamma_{\delta}>0$ such that $\left|\dot{\Delta}_{o}\left(\lambda_{n}^{0}\right)\right| \geq \gamma_{\delta}>0$ is satisfied for all $n$. We also have

$$
\lambda_{n}^{0}=n+\frac{1}{\pi} \beta^{+}(\pi)+h_{n}
$$

where $\sup _{n}\left|h_{n}\right| \leq M$ for some constant $M>0$ [40] (see also [41]). Further, substituting (53) into (52) after certain transformations [1, page 67], we reach $\varepsilon_{n} \in l_{2}$. We can obtain more precisely

$$
\begin{aligned}
& \varepsilon_{n}=\frac{1}{2 \dot{\Delta}_{o}\left(\lambda_{n}^{0}\right) \lambda_{n}^{0}}\left\{\left[\alpha^{+} \sin \left(\lambda_{n}^{0} \pi-\beta_{1}(\pi)\right)\right.\right. \\
& \left.+\alpha^{-} \sin \left(\lambda_{n}^{0}(2 a-\pi)+\beta_{2}(\pi)\right)\right] \\
& \times \int_{0}^{\pi}\left(q(x)+p^{2}(x)\right) d x \\
& -\left[\alpha^{+} \cos \left(\lambda_{n}^{0} \pi-\beta_{1}(\pi)\right)\right. \\
& \left.+\alpha^{-} \cos \left(\lambda_{n}^{0}(2 a-\pi)+\beta_{2}(\pi)\right)\right] \\
& \times(p(\pi)-p(0))\} \\
& +\frac{1}{\dot{\Delta}_{o}\left(\lambda_{n}^{0}\right) \lambda_{n}^{0}}\left[\int_{0}^{\pi} B_{t}^{\prime}(\pi, t) \cos \lambda_{n}^{0} t d t\right. \\
& \left.-\int_{0}^{\pi} A_{t}^{\prime}(\pi, t) \sin \lambda_{n}^{0} t d t\right] \\
& +\frac{o\left(\varepsilon_{n}\right)}{\lambda_{n}^{0}}, \quad n \longrightarrow \infty \text {. }
\end{aligned}
$$


Since $\int_{0}^{\pi} B_{t}^{\prime}(\pi, t) \cos \lambda_{n}^{0} t d t \in l_{2}, \int_{0}^{\pi} A_{t}^{\prime}(\pi, t) \sin \lambda_{n}^{0} t d t \in$ $l_{2}$, we have

$$
\begin{aligned}
\varepsilon_{n}=\frac{1}{2 \dot{\Delta}_{o}\left(\lambda_{n}^{0}\right) \lambda_{n}^{0}\{}\{ & \alpha^{+} \sin \left(\lambda_{n}^{0} \pi-\beta_{1}(\pi)\right) \\
& \left.+\alpha^{-} \sin \left(\lambda_{n}^{0}(2 a-\pi)+\beta_{2}(\pi)\right)\right] \\
& \times \int_{0}^{\pi}\left(q(x)+p^{2}(x)\right) d x \\
- & {\left[\alpha^{+} \cos \left(\lambda_{n}^{0} \pi-\beta_{1}(\pi)\right)\right.} \\
& \left.+\alpha^{-} \cos \left(\lambda_{n}^{0}(2 a-\pi)+\beta_{2}(\pi)\right)\right] \\
& \times(p(\pi)-p(0))\}+\frac{\delta_{n}}{\lambda_{n}^{0}},
\end{aligned}
$$

where $\delta_{n} \in l_{2}$. Hence we obtain

$$
\lambda_{n}=\lambda_{n}^{0}+\frac{d_{n}}{\lambda_{n}^{0}}+\frac{\delta_{n}}{\lambda_{n}^{0}},
$$

where

$$
\begin{aligned}
d_{n}=\frac{1}{2 \dot{\Delta}_{o}\left(\lambda_{n}^{0}\right)}\{[ & \alpha^{+} \sin \left(\lambda_{n}^{0} \pi-\beta^{+}(\pi)\right) \\
& \left.+\alpha^{-} \sin \left(\lambda_{n}^{0}(2 a-\pi)+\beta^{-}(\pi)\right)\right] \\
& \times \int_{0}^{\pi}\left(q(x)+p^{2}(x)\right) d x \\
- & {\left[\alpha^{+} \cos \left(\lambda_{n}^{0} \pi-\beta^{+}(\pi)\right)\right.} \\
& \left.+\alpha^{-} \cos \left(\lambda_{n}^{0}(2 a-\pi)+\beta^{-}(\pi)\right)\right] \\
& \times(p(\pi)-p(0))\}
\end{aligned}
$$

is a bounded sequence. The proof is completed.

Lemma 13. Normalizing numbers $\alpha_{n}$ of the problem $L(\alpha, a)$ are positive and the formula

$$
\alpha_{n}=\frac{\pi}{2}\left[\left(\alpha^{+}\right)^{2}+\left(\alpha^{-}\right)^{2}\right]+\frac{d_{11}}{\lambda_{n}^{0}}+\frac{d_{1 n}}{n}
$$

holds, where $d_{11}=-(\alpha \pi / 2) p(0), d_{1 n} \in l_{2}$.

Proof. The formula (58) can be easily obtained from the equalities

$$
\begin{aligned}
& A(x, x) \sin \lambda_{n} x-B(x, x) \cos \lambda_{n} x \\
& =\frac{\alpha^{+}}{2}\left\{(p(x)-p(0)) \cos \left(\lambda_{n} x-\beta^{+}(x)\right)\right. \\
& \left.\quad+\int_{0}^{x}\left(q(t)+p^{2}(t)\right) d t \sin \left(\lambda_{n} x-\beta^{+}(x)\right)\right\},
\end{aligned}
$$

$$
\begin{gathered}
{[A(x, 2 a-x+0)-A(x, 2 a-x-0)] \sin \lambda_{n}(2 a-x)} \\
-[B(x, 2 a-x+0)-B(x, 2 a-x-0)] \cos \lambda_{n}(2 a-x) \\
=\frac{\alpha^{-}}{2}\left\{\int_{0}^{x}\left(q(t)+p^{2}(t)\right) d t \sin \left(\lambda_{n}(2 a-x)+\beta^{-}(x)\right)\right. \\
\left.-(p(x)-p(0)) \cos \left(\lambda_{n}(2 a-x)+\beta^{-}(x)\right)\right\}
\end{gathered}
$$

by using the asymptotic formula (49) for $\lambda_{n}$.

\section{Inverse Problems}

Together with $L(\alpha, a)$, we consider the boundary value problem $\widetilde{L}(\alpha, a)$ of the same form but with different coefficients $(\widetilde{q}, \widetilde{p}, \widetilde{\alpha}, \widetilde{a})$. It is assumed in what follows that if a certain symbol $\gamma$ denotes an object related to the problem $L(\alpha, a)$, then $\tilde{\gamma}$ will denote the corresponding object related to the problem $\widetilde{L}(\alpha, a)$.

In the present section, we investigate some inverse spectral problem of the reconstruction of a boundary value problem $L(\alpha, a)$ of type (1)-(4) from its spectral characteristics. Namely, we consider the inverse problems of reconstruction of the boundary value problem $L(\alpha, a)$ from the Weyl function, from the spectral data $\left\{\lambda_{n}, \alpha_{n}\right\}_{n \geq 0}$, and from two spectra $\left\{\lambda_{n}, \mu_{n}\right\}_{n \geq 0}$ and prove that the following two lemmas can be easily obtained from asymptotic behavior (49) of the eigenvalues $\lambda_{n}$.

Lemma 14. If $\lambda_{n}=\tilde{\lambda}_{n}, n=0, \pm 1, \pm 2, \ldots$, then $\beta^{+}(\pi)=$ $\widetilde{\beta}^{+}(\pi), \beta^{-}(\pi)=\widetilde{\beta}^{-}(\pi)$, that is, the sequence $\left\{\lambda_{n}\right\}$ uniquely determines $\beta^{ \pm}(\pi)$.

Lemma 15. If $\lambda_{n}=\widetilde{\lambda}_{n}, n=0, \pm 1, \pm 2, \ldots$, then $a=\tilde{a}, \alpha=\widetilde{\alpha}$, that is, the sequence $\left\{\lambda_{n}\right\}$ uniquely determines numbers $a$ and $\alpha$.

Let $\Phi(x, \lambda)$ be the solution of (1) under the conditions $U(\Phi)=1, V(\Phi)=0$ and under the jump conditions (3). One sets $M(\lambda):=\Phi(0, \lambda)$. The functions $\Phi(x, \lambda)$ and $M(\lambda)$ are called the Weyl solution and Weyl function for the boundary value problem $L(\alpha, a)$, respectively. Using the solution $\varphi(x, \lambda)$ defined in the previous sections one has

$$
\begin{array}{r}
\Phi(x, \lambda)=-\frac{\psi(x, \lambda)}{\Delta(\lambda)}=S(x, \lambda)+M(\lambda) \varphi(x, \lambda), \\
M(\lambda)=-\frac{\psi(0, \lambda)}{\Delta(\lambda)},
\end{array}
$$

where $\psi(x, \lambda)$ is a solution of (1) satisfying the conditions $\psi(\pi, \lambda)=0, \psi^{\prime}(\pi, \lambda)=-1$, and the jump conditions (3), and $S(x, \lambda)$ is defined from the equality

$$
\psi(x, \lambda)=\psi(0, \lambda) \varphi(x, \lambda)-\Delta(\lambda) S(x, \lambda) .
$$


Note that, by virtue of equalities $\langle\varphi(x, \lambda), S(x, \lambda)\rangle \equiv 1$ and (60), one has

$$
\begin{gathered}
\langle\Phi(x, \lambda), \varphi(x, \lambda)\rangle \equiv 1, \\
\langle\varphi(x, \lambda), \psi(x, \lambda)\rangle \equiv-\Delta(\lambda) \quad \text { for } x \neq a .
\end{gathered}
$$

The following theorem shows that the Weyl function uniquely determines the potentials and the coefficients of the boundary value problem $L(\alpha, a)$.

Theorem 16. If $M(\lambda)=\widetilde{M}(\lambda)$, then $L(\alpha, a)=\widetilde{L}(\alpha, a)$. Thus, the boundary value problem $L(\alpha, a)$ is uniquely defined by the Weyl function.

Proof. Since

$$
\psi^{(\nu)}(x, \lambda)=O\left(|\lambda|^{\nu-1} \exp (|\operatorname{Im} \lambda|(\pi-x))\right), \quad \lambda \in \widetilde{G}_{\delta},
$$

$$
|\Delta(\lambda)| \geq C_{\delta} \exp (|\operatorname{Im} \lambda| \pi), \quad \lambda \in \widetilde{G}_{\delta}, C_{\delta}<0, \nu=0,1,
$$

it is easy to observe that

$$
\left|\Phi^{(\nu)}(x, \lambda)\right| \leq C_{\delta}|\lambda|^{\nu-1} \exp (-|\operatorname{Im} \lambda| x), \quad \lambda \in G_{\delta} .
$$

Let us define the matrix $P(x, \lambda)=\left[P_{j k}(x, \lambda)\right]_{j, k=1,2}$, where

$$
\begin{aligned}
& P_{j 1}(x, \lambda)=\varphi^{(j-1)}(x, \lambda) \widetilde{\Phi}^{\prime}(x, \lambda)-\Phi^{(j-1)}(x, \lambda) \widetilde{\varphi}^{\prime}(x, \lambda), \\
& P_{j 2}(x, \lambda)=\Phi^{(j-1)}(x, \lambda) \widetilde{\varphi}^{\prime}(x, \lambda)-\varphi^{(j-1)}(x, \lambda) \widetilde{\Phi}(x, \lambda) .
\end{aligned}
$$

Then we have

$$
\begin{aligned}
& \varphi(x, \lambda)=P_{11}(x, \lambda) \widetilde{\varphi}(x, \lambda)+P_{12}(x, \lambda) \widetilde{\varphi}^{\prime}(x, \lambda), \\
& \Phi(x, \lambda)=P_{11}(x, \lambda) \widetilde{\Phi}(x, \lambda)+P_{12}(x, \lambda) \widetilde{\Phi}^{\prime}(x, \lambda) .
\end{aligned}
$$

According to (60) and (65), for each fixed $x$, the functions $P_{j k}(x, \lambda)$ are meromorphic in $\lambda$ with poles at points $\lambda_{n}$ and $\tilde{\lambda}_{n}$. Denote $G_{\delta}^{\circ}=G_{\delta} \cap \widetilde{G}_{\delta}$. By virtue of (65), (66), and

$$
\varphi^{(\nu)}(x, \lambda)=O\left(|\lambda|^{\nu} \exp (|\operatorname{Im} \lambda| x)\right), \quad \lambda \in G_{\delta}^{\circ},
$$

we get

$$
\left|P_{12}(x, \lambda)\right| \leq C_{\delta}|\lambda|^{-1}, \quad\left|P_{11}(x, \lambda)\right| \leq C_{\delta}, \quad \lambda \in G_{\delta}^{\circ} .
$$

It follows from (60) and (66) that if $M(\lambda) \equiv \widetilde{M}(\lambda)$, then for each fixed $x$ the functions $P_{1 k}(x, \lambda)$ are entire in $k$. Together with (69) this yields $P_{12}(x, \lambda) \equiv 0, P_{12}(x, \lambda) \equiv A(x)$. Now using (67), we obtain

$$
\varphi(x, \lambda) \equiv A(x) \widetilde{\varphi}(x, \lambda), \quad \Phi(x, \lambda) \equiv A(x) \widetilde{\Phi}(x, \lambda) .
$$

Therefore, for $|\lambda| \rightarrow \infty$, arg $\lambda \in[\varepsilon, \pi-\varepsilon](\varepsilon>0)$, we have

$$
\varphi(x, \lambda)=\frac{b}{2} \exp \left(-i\left(\lambda x-\beta_{1}(x)\right)\right)\left(1+O\left(\frac{1}{\lambda}\right)\right)
$$

where $b=1$ for $x<a$ and $b=\alpha^{+}$for $x>a$. Similarly, one can calculate

$$
\begin{array}{r}
\Phi(x, \lambda)=(i \lambda b)^{-1} \exp \left(i\left(\lambda x-\beta_{1}(x)\right)\right)\left(1+O\left(\frac{1}{\lambda}\right)\right), \\
|\lambda| \longrightarrow \infty, \arg \lambda \in[\varepsilon, \pi-\varepsilon] .
\end{array}
$$

Finally, taking into account the relations $\langle\Phi(x, \lambda), \varphi(x, \lambda)\rangle \equiv$ 1 and (65), we have $\alpha^{+}=\widetilde{\alpha}^{+}, A(x) \equiv 1$, that is, $\varphi(x, \lambda) \equiv$ $\widetilde{\varphi}(x, \lambda), \Phi(x, \lambda) \equiv \widetilde{\Phi}(x, \lambda)$ for all $x$ and $\lambda$. Consequently, $L(\alpha, a)=\widetilde{L}(\alpha, a)$. The theorem is proved.

The following two theorems show that two spectra and spectral data also uniquely determine the potentials and the coefficients of the boundary value problem $L(\alpha, a)$.

Theorem 17. If $\lambda_{n}=\tilde{\lambda}_{n}, \mu_{n}=\tilde{\mu}_{n}, n=0, \pm 1, \pm 2, \ldots$, then $L(\alpha, a)=\widetilde{L}(\alpha, a)$.

Proof. It is obvious that characteristic functions $\Delta(\lambda)$ and $\psi(0, \lambda)$ are uniquely determined by the sequences $\left\{\lambda_{n}^{2}\right\}$ and $\left\{\mu_{n}^{2}\right\}(n=0, \pm 1, \pm 2, \ldots)$, respectively. If $\lambda_{n}=\tilde{\lambda}_{n}, \mu_{n}=$ $\tilde{\mu}_{n}, n=0, \pm 1, \pm 2, \ldots$, then $\Delta(\lambda) \equiv \widetilde{\Delta}(\lambda), \psi(0, \lambda) \equiv \widetilde{\psi}(0, \lambda)$. It follows from $(60)$ that $M(\lambda) \equiv \widetilde{M}(\lambda)$. Therefore, applying Theorem 16, we conclude that $L(\alpha, a)=\widetilde{L}(\alpha, a)$. The proof is completed.

Theorem 18. If $\lambda_{n}=\tilde{\lambda}_{n}, \alpha_{n}=\widetilde{\alpha}_{n}, n=0, \pm 1, \pm 2, \ldots$, then $L(\alpha, a)=\widetilde{L}(\alpha, a)$, that is, spectral data $\left\{\lambda_{n}, \alpha_{n}\right\}$ uniquely determines the problem $L(\alpha, a)$.

Proof. It is obvious that the Weyl function $M(\lambda)$ is meromorphic with simple poles at points $\lambda_{n}^{2}$. Using the expression

$$
\Delta(\lambda)=\Delta_{0}(\lambda)+\int_{0}^{\pi} A(\pi, \lambda) \cos \lambda t d t+\int_{0}^{\pi} B(\pi, \lambda) \sin \lambda t d t
$$

and equalities $2 \lambda_{n} \beta_{n} \alpha_{n}=-\dot{\Delta}\left(\lambda_{n}\right), \psi\left(x, \lambda_{n}\right)=\beta_{n} \varphi\left(x, \lambda_{n}\right)$, we have

$$
\operatorname{Re}_{\lambda=\lambda_{n}} M(\lambda)=-\frac{\psi\left(0, \lambda_{n}\right)}{\dot{\Delta}\left(\lambda_{n}\right)}=-\frac{\beta_{n}}{\dot{\Delta}\left(\lambda_{n}\right)}=\frac{1}{2 \lambda_{n} \alpha_{n}} .
$$

Since the Weyl function $M(\lambda)$ is regular for $\lambda \in \Gamma_{n}$, applying the Rouche theorem, we conclude that

$$
M(\lambda)=\frac{1}{2 \pi i} \int_{\Gamma_{n}} \frac{M(\mu)}{\mu-\lambda} d \mu, \quad \lambda \in \operatorname{int} \Gamma_{n} .
$$

Taking (60) and (63) into account, we arrive at $|M(\lambda)| \leq$ $C_{\delta}|\lambda|^{-1}, \lambda \in G_{\delta}$. Therefore

$$
M(\lambda)=\lim _{n \rightarrow \infty} \frac{1}{2 \pi i} \int_{\Gamma_{n 1}} \frac{M(\mu)}{\mu-\lambda} d \mu,
$$

where $\Gamma_{n 1}=\left\{\lambda:|\lambda|=\left|\lambda_{n}^{0}\right|\right\}, n=0, \pm 1, \pm 2, \ldots$. Hence, by the residue theorem, we have

$$
M(\lambda)=\sum_{n=-\infty}^{\infty} \frac{1}{2 \lambda_{n} \alpha_{n}\left(\lambda-\lambda_{n}\right)} .
$$


Finally, from the equality $M(\lambda) \equiv \widetilde{M}(\lambda)$, applying Theorem 16, we conclude that $L(\alpha, a)=\widetilde{L}(\alpha, a)$. The theorem is proved.

\section{References}

[1] V. A. Marchenko, Sturm-Liouville Operators and Applications, vol. 22 of Operator Theory: Advances and Applications, Birkhäuser, Basel, Switzerlands, 1986.

[2] B. M. Levitan, Inverse Sturm-Liouville Problems, Nauka, Moscow, Russia, 1987.

[3] R. Beals, P. Deift, and C. Tomei, Direct and Inverse Scattering on the Line, vol. 28 of Mathematical Surveys and Monographs, American Mathematical Society, Providence, RI, USA, 1988.

[4] V. A. Yurko, Introduction to the Theory of Inverse Spectral Problems, Fizmatlit, Moscow, Russia, 2007, English translation, Inverse Spectral Problems for Differential Operators and Their Applications, Gordon and Breach, Amsterdam, The Netherlands, 2000.

[5] B. M. Levitan and M. G. Gasymov, "Determination of a differential equation by two spectra," Uspekhi Matematicheskikh Nauk, vol. 19, no. 2, pp. 3-63, 1964.

[6] M. Jaulent and C. Jean, "The inverse problem for the onedimensional Schrödinger equation with an energy-dependent potential. I, II," Annales de l'Institut Henri Poincaré A, vol. 25, no. 2, pp. 105-137, 1976.

[7] M. G. Gasymow, "On the spectral theory of dierential operators polnomially depending on a parameter," Uspekhi Matematicheskikh Nauk, vol. 37, no. 4, p. 99, 1982 (Russian).

[8] V. A. Yurko, "An inverse problem for differential operator pencils," Matematicheskiu Sbornik, vol. 191, no. 10, pp. 137-160, 2000, English translation, Sbornik: Mathematics vol. 191, no. 10, pp. 1561-1586, 2000.

[9] M. G. Gasymov and G. Š. Guseřnov, "Determination of a diffusion operator from spectral data," Akademiya Nauk Azerbă̌dzhanskoĭ SSR. Doklady, vol. 37, no. 2, pp. 19-23, 1981 (Russian).

[10] G. Sh. Guseinov, "On the spectral analysis of a quadratic pencil of Sturm-Liouville operators," Doklady Akademii Nauk SSSR, vol. 285, no. 6, pp. 1292-1296, 1985, English translation, Soviet Mathematics Doklady, vol. 32, no.3, pp. 859-862, 1985.

[11] G. Sh. Guseĭnov, "Inverse spectral problems for a quadratic pencil of Sturm-Liouville operators on a finite interval," in Spectral Theory of Operators and Its Applications, pp. 51-101, Èlm, Baku, Azerbaijan, 1986.

[12] I. M. Nabiev, "Inverse periodic problem for a diffusion operator," Transactions of Academy of Sciences of Azerbaijan, vol. 23, no. 4, pp. 125-130, 2003.

[13] I. M. Nabiev, "The inverse spectral problem for the diffusion operator on an interval," Matematicheskaya Fizika, Analiz, Geometriya, vol. 11, no. 3, pp. 302-313, 2004 (Russian).

[14] V. N. Pivovarchik, "Reconstruction of the potential of the Sturm-Liouville equation from three spectra of boundary value problems," Funktsional'nyi Analiz i ego Prilozheniya, vol. 33, no. 3, pp. 87-90, 1999, English translation, Functional Analysis and Its Applications, vol. 33, no. 3, pp. 233-235, 1999.

[15] V. P. Meshonav and A. L. Feldstein, Automatic Design of Directional Couplers, Sviaz, Moscow, Russia, 1980.

[16] O. N. Litvinenko and V. I. Soshnikov, The Theory of Heterogeneous Lines and Their Applications in Radio Engineering, Radio, Moscow, Russia, 1964.
[17] R. J. Krueger, "Inverse problems for nonabsorbing media with discontinuous material properties," Journal of Mathematical Physics, vol. 23, no. 3, pp. 396-404, 1982.

[18] D. G. Shepelsky, “The inverse problem of reconstruction of the medium's conductivity in a class of discontinuous and increasing functions," Advances in Soviet Mathematics, vol. 19, pp. 303-309, 1997.

[19] F. R. Lapwood and T. Usami, Free Oscillation of the Earth, Cambridge University Press, Cambridge, UK, 1981.

[20] G. Borg, "Eine Umkehrung der Sturm-Liouvilleschen Eigenwertaufgabe," Acta Mathematica, vol. 78, pp. 1-96, 1946.

[21] B. M. Levitan and I. S. Sargsyan, Introduction To Spectral Theory, vol. 39 of Translations of Mathematical Monographs, American Mathematical Society, Providence, RI, USA, 1975.

[22] J. Pöschel and E. Trubowitz, Inverse Spectral Theory, vol. 130 of Pure and Applied Mathematics, Academic Press, Boston, Mass, USA, 1987.

[23] V. A. Yurko, Inverse spectral problems for differential operators and their applications, vol. 2 of Analytical Methods and Special Functions, Gordon and Breach, Amsterdam, The Netherlands, 2000.

[24] G. Freiling and V. Yurko, Inverse Sturm-Liouville problems and their applications, Nova Science, Huntington, NY, USA, 2001.

[25] J. R. McLaughlin, "Analytical methods for recovering coefficients in differential equations from spectral data," SIAM Review, vol. 28, no. 1, pp. 53-72, 1986.

[26] O. H. Hald, "Discontinuous inverse eigenvalue problems," Communications on Pure and Applied Mathematics, vol. 37, no. 5, pp. 539-577, 1984.

[27] A. McNabb, R. S. Anderssen, and E. R. Lapwood, "Asymptotic behavior of the eigenvalues of a Sturm-Liouville system with discontinuous coefficients," Journal of Mathematical Analysis and Applications, vol. 54, no. 3, pp. 741-751, 1976.

[28] W. W. Symes, "Impedance profile inversion via the first transport equation," Journal of Mathematical Analysis and Applications, vol. 94, no. 2, pp. 435-453, 1983.

[29] T. Aktosun, M. Klaus, and C. van der Mee, "Inverse wave scattering with discontinuous wave speed," Journal of Mathematical Physics, vol. 36, no. 6, pp. 2880-2928, 1995.

[30] W. Eberhard, G. Freiling, and A. Schneider, "On the distribution of the eigenvalues of a class of indefinite eigenvalue problems," Differential and Integral Equations, vol. 3, no. 6, pp. 1167-1179, 1990.

[31] R. Carlson, "An inverse spectral problem for Sturm-Liouville operators with discontinuous coefficients," Proceedings of the American Mathematical Society, vol. 120, no. 2, pp. 475-484, 1994.

[32] R. Kh. Amirov, "On Sturm-Liouville operators with discontinuity conditions inside an interval," Journal of Mathematical Analysis and Applications, vol. 317, no. 1, pp. 163-176, 2006.

[33] V. A. Yurko, "On higher-order differential operators with a singular point," Inverse Problems, vol. 9, no. 4, pp. 495-502, 1993.

[34] V. A. Yurko, "On higher-order differential operators with a regular singularity," Matematicheskiu Sbornik, vol. 186, no. 6, pp. 133-160, 1995, English translation, Sbornik: Mathematics, vol. 186, no. 6, pp. 901-928, 1995.

[35] V. Yurko, "Integral transforms connected with differential operators having singularities inside the interval," Integral Transforms and Special Functions, vol. 5, no. 3-4, pp. 309-322, 1997. 
[36] R. Kh. Amirov and V. A. Yurko, "On differential operators with a singularity and discontinuity conditions inside an interval," Ukrainian Mathematical Journal, vol. 53, no. 11, pp. 1443-1457, 2001.

[37] G. Freiling and V. Yurko, "Inverse spectral problems for singular non-selfadjoint differential operators with discontinuities in an interior point," Inverse Problems, vol. 18, no. 3, pp. 757-773, 2002.

[38] R. Bellman and K. L. Cooke, Differential-Difference Equations, Academic Press, New York, NY, USA, 1963.

[39] B. Ya. Levin, Entire Functions, MGV, Moscow, Russia, 1971.

[40] B. F. Jdanovich, "Formulae for the zeros of Drichlet polynomials and quasi-polynomials," Doklady Akademii Nauk SSSR, vol. 135, no. 8, pp. 1046-1049, 1960.

[41] M. Kreĭn and B. Ya. Levin, "On entire almost periodic functions of exponential type," Doklady Akademii Nauk SSSR, vol. 64, pp. 285-287, 1949. 


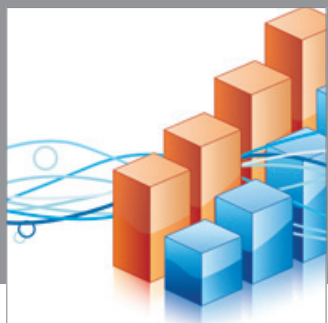

Advances in

Operations Research

mansans

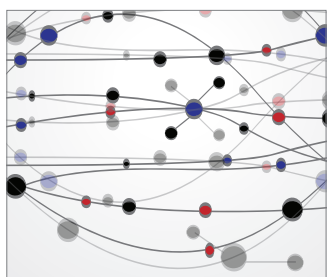

The Scientific World Journal
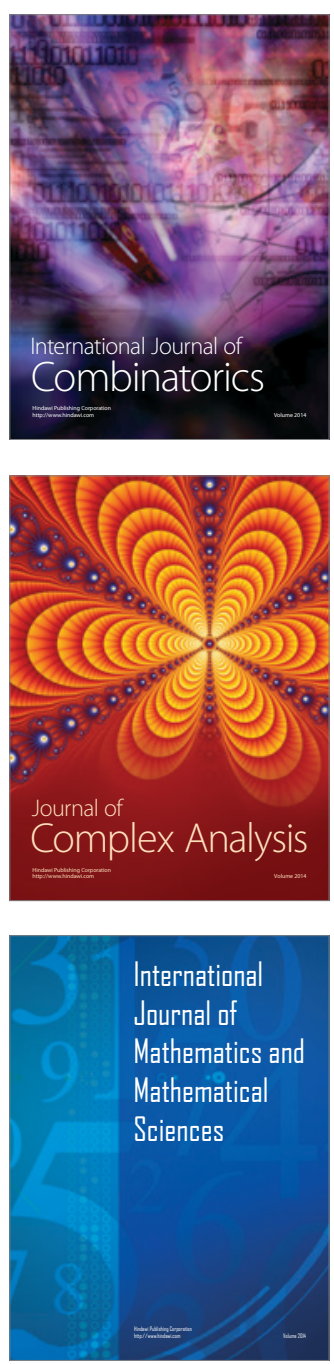
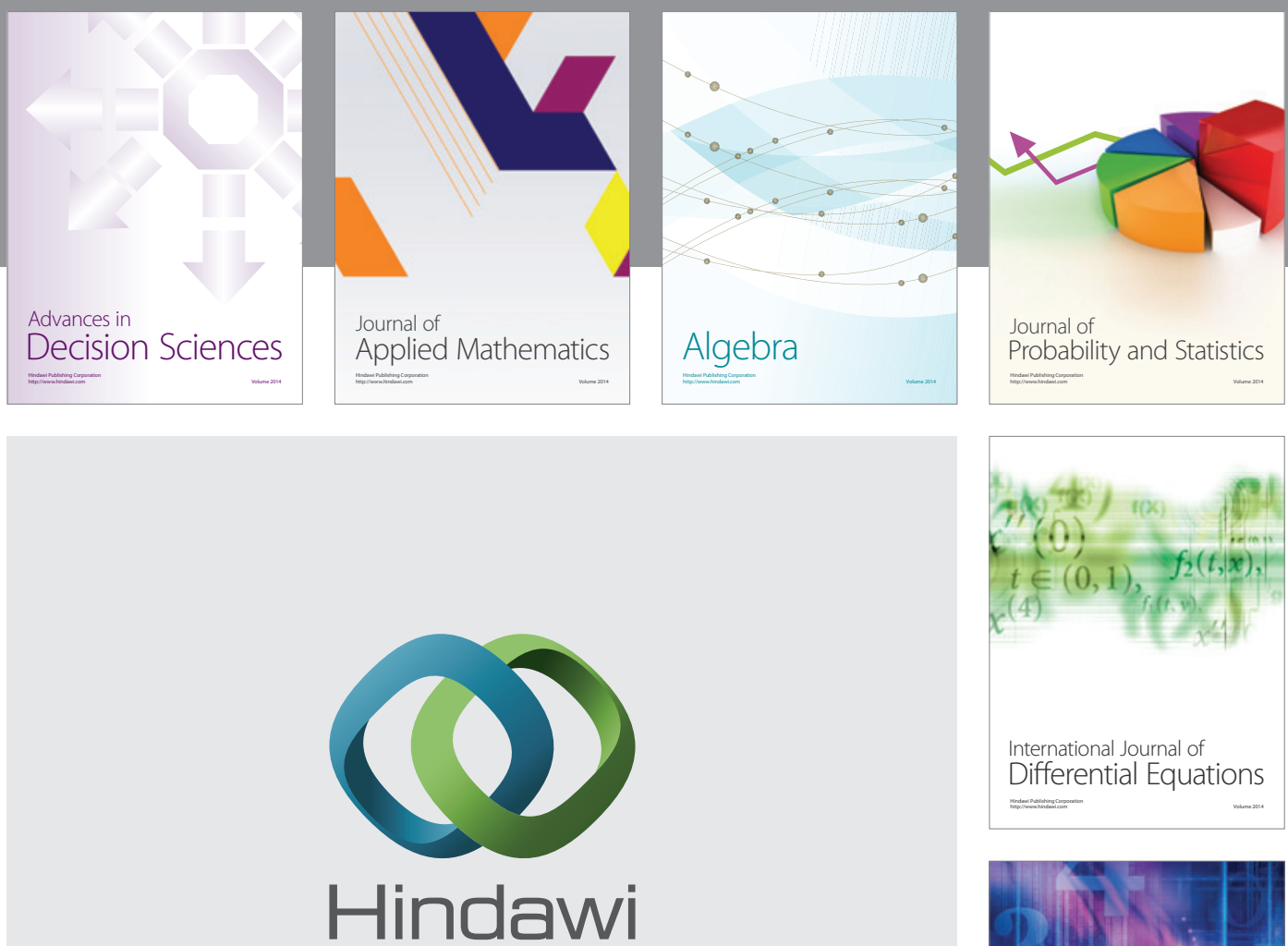

Submit your manuscripts at http://www.hindawi.com
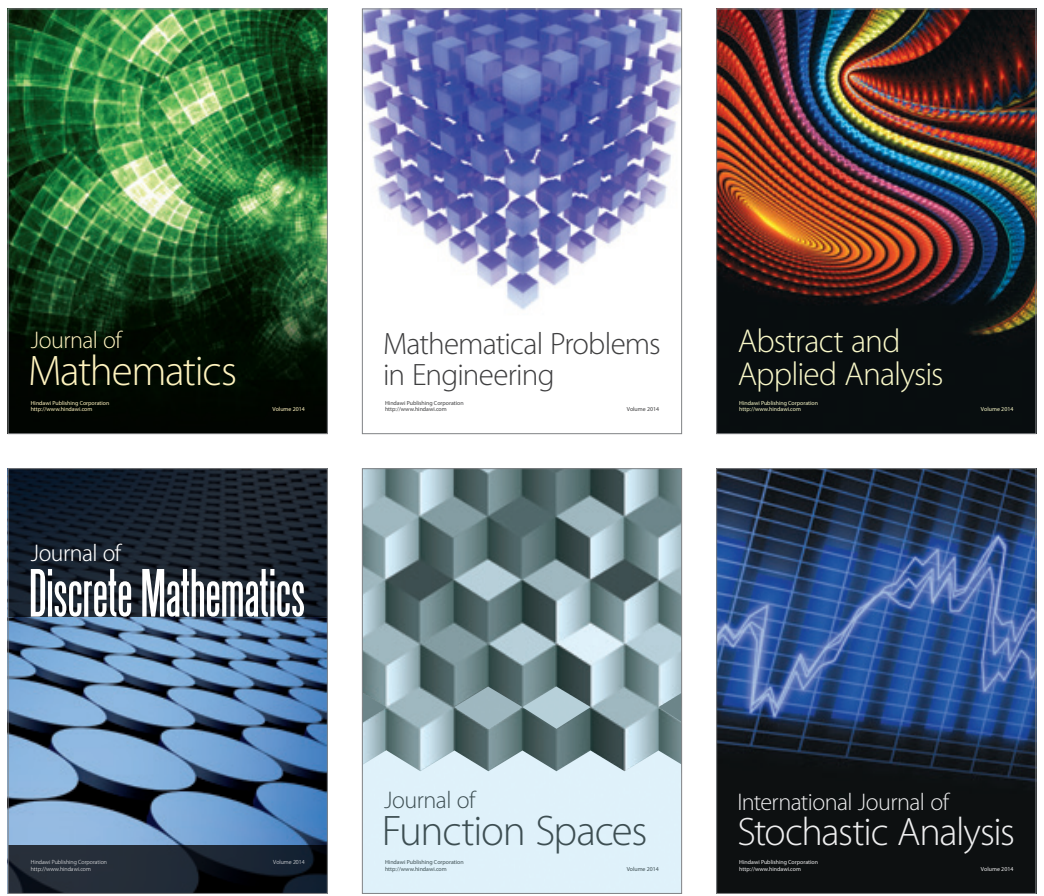

Journal of

Function Spaces

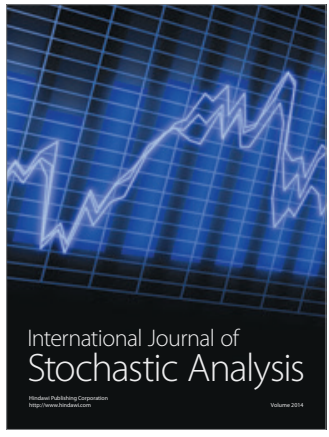

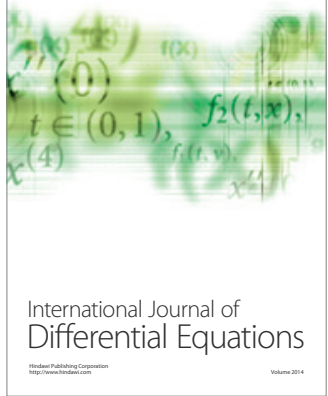
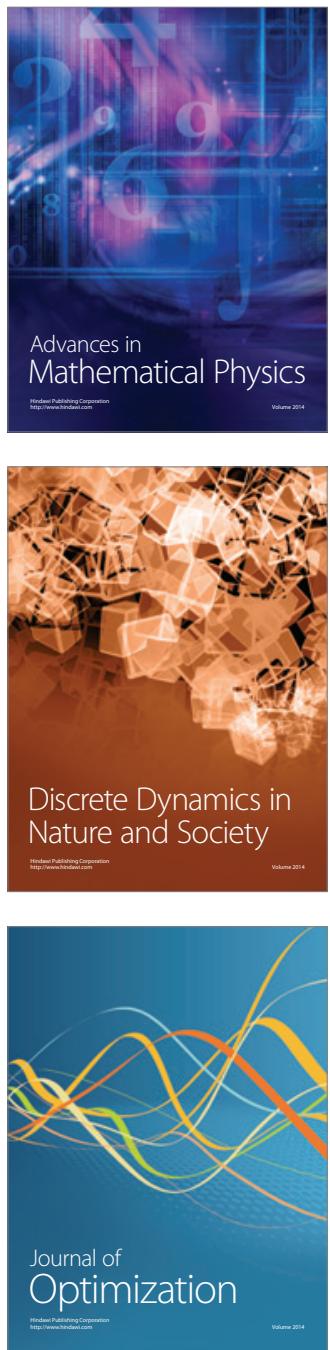